\title{
Silencing HIWI suppresses the growth, invasion and migration of glioma cells
}

\author{
XIUYU WANG $^{1 *}$, XIAOGUANG TONG ${ }^{2 *}$, HONGMEI GAO $^{3 *}$, XIAOLING YAN ${ }^{2}$, \\ XINNV XU ${ }^{3}$, SHUPENG SUN ${ }^{2}$, QIONG WANG ${ }^{2}$ and JINHUAN WANG ${ }^{2}$ \\ ${ }^{1}$ The Graduate School, Tianjin Medical University, Tianjin 300070; ${ }^{2}$ Tianjin Neurosurgery Institute, \\ Tianjin Cerebral Vascular and Neural Degenerative Disease Key Laboratory, Tianjin Huanhu Hospital, \\ Tianjin 300060; ${ }^{3}$ Department of Critical Care Medicine, Tianjin First Central Hospital, Tianjin 300192, P.R. China
}

Received July 13, 2014; Accepted September 2, 2014

DOI: $10.3892 /$ ijo.2014.2673

\begin{abstract}
The HIWI gene is one of the members of the PIWI gene family that is important for stem cell self-renewal and expressed highly in certain human tumors. Some studies have demonstrated that HIWI plays a key role in the development of tumors in cervical, colon and liver cancer. Previous studies have demonstrated that HIWI is associated with prognosis of patients with glioma. However, there is no report on the analysis of HIWI in the biological characteristics of glioma cells. The aim of the study was to investigate whether HIWI plays an important role in the progress of glioma. Silencing HIWI inhibited cell proliferation by promoting apoptosis and increased cell cycle arrest. The expression of proteins related to apoptosis and the cell cycle, including p21, cyclin D1, Bcl-2, and Bax was significantly altered. Moreover, knockdown of HIWI inhibited the migration and invasion of glioma cells by reducing the expression of MMP-2 and MMP-9. Furthermore, we found that reduction of HIWI inhibited tumor growth in vivo. These findings suggest that HIWI is an oncogene involved in the progression of glioma.
\end{abstract}

\section{Introduction}

Glioblastoma multiforme (GBM) is the most common primary intracranial tumor in adults, with a combined incidence of 5-8 per 100,000 individuals. It is highly aggressive, with a poor prognosis $(1,2)$. Although the treatment of GBM has advanced, the median survival of patients with GBM is generally only

Correspondence to: Professor Jinhuan Wang or Professor Qiong Wang, Tianjin Neurosurgery Institute, Tianjin Cerebral Vascular and Neural Degenerative Disease Key Laboratory, Tianjin Huanhu Hospital, 122 Qixiangtai Street, Tianjin 300060, P.R. China

E-mail: wangjinhuanfch@126.com

E-mail: lailwq@126.com

*Contributed equally

Key words: glioma, HIWI, cell proliferation, invasion, migration
9-12 months (3). At present, GBM treatments include surgery, radiotherapy, and chemotherapy. However, these treatments frequently fail: i) due to its highly invasive nature, operations cannot remove tumor tissue thoroughly; and ii) tumors develop resistance to radiotherapy and chemotherapy. Therefore, it is critical to develop more effective therapies based on novel molecular targets (4) and to identify novel therapeutic strategies to improve prognosis for patients with GBM.

Cox et al (5) first discovered PIWI in a genetic screen for mutants that affected the asymmetrical division of stem cells in the Drosophila germline. Early studies revealed that Drosophila PIWI is an important factor for gametogenesis, and a key regulator of female germline stem cells. The PIWI protein family is highly conserved in many species, including humans. To date, four PIWI proteins have been reported in humans: PIWIL1/HIWI, PIWIL2/HILI, PIWIL3, and PIWIL4/HIWI2 (6). HIWI (also named PIWIL1), a human homologue of the PIWI family, plays an important role in the self-renewal of stem cells (7). Tumor, germ, and stem cells all share common characteristics, for example the ability for rapid and infinite self-renewal. Therefore, it is not surprising that HIWI has been implicated in oncogenesis. Several studies have demonstrated that HIWI is expressed in tumor tissues, and is one of the most important elements in the regulation of cancer cells $(8,9)$. HIWI was also correlated with patient prognosis in pancreatic, colon, colorectal, lung, and endometrial cancer (10-14).

Sun et al (15) reported that HIWI was overexpressed in glioma tissues and cell lines, and associated the prognosis of patients with glioma. However, it remains unclear whether HIWI plays an important role in the tumorigenesis and development of GBM. Therefore, the aim of the present study was to investigate the effects of HIWI in aspects of GBM including proliferation, invasion, and migration, and to define the corresponding molecular mechanisms using RNA interference and flow cytometry analysis.

\section{Materials and methods}

Cell culture. The human glioblastoma cell lines A172 and U251 were purchased from the Peking Union Medical College cell library (Beijing, China). Cells were cultured 
in Dulbecco's modified Eagle's medium (DMEM) supplemented with penicillin/streptomycin and $10 \%$ fetal bovine serum (FBS) in a humidified atmosphere containing $5 \% \mathrm{CO}_{2}$ at $37^{\circ} \mathrm{C}$.

Transfection. Small interfering RNA against HIWI and a negative control (scrambled control) were purchased from GenePharma (Shanghai, China). The following siRNA sequences described previously (16) were used: HIWI, 5'-GCC GUUCAUACAAGACUAATT-3'; negative control, 5'-UUC UCCGAACGUGUCACGUTT-3'. Transfection was performed using Lipofectamine 2000 Reagent (Invitrogen Life Technologies, Carlsbad, CA, USA) according to the manufacturer's instructions. In brief, A172 or U251 cells were seeded into 6- or 96-well plates (Corning Costar, Cambridge, MA, USA). The following day (when the cells were $\sim 40 \%$ confluent), the culture media were aspirated, and the cell monolayer was washed with pre-warmed sterile phosphate-buffered saline (PBS). Transfection complexes were prepared following the manufacturer's instructions.

Western blotting. U251 and A172 control and treated cells were washed twice in ice-cold PBS, and lysed in M-PER Mammalian Protein Extraction Reagent (CW Biotech, Beijing, China) containing protease and phosphatase inhibitor cocktails. Lysates were centrifuged at $12,000 \mathrm{x}$ g for $15 \mathrm{~min}$ at $4^{\circ} \mathrm{C}$. The supernatants were then added to an equal volume of sample buffer (50 mM Tris- $\mathrm{HCl}, 4 \%$ SDS, $0.01 \%$ bromophenol blue, $20 \%$ glycerol, and 2\% 2-Mercaptoethanol, $\mathrm{pH}$ 6.8), heat-denatured, and placed on ice for $5 \mathrm{~min}$. Protein concentrations were determined using the BCA Protein Assay kit (CW Biotech). Equal amounts of protein were loaded onto $12 \%$ Tris-glycine SDS-PAGE gels, and separated at $80-120 \mathrm{~V}$ for $1.5-2 \mathrm{~h}$. The proteins were transferred to nitrocellulose membrane, and blocked with $5 \%$ milk in TBST buffer. Membranes were then incubated with primary antibodies at $4^{\circ} \mathrm{C}$ overnight. After washing three times in TBST buffer for 5 min each, membranes were incubated with secondary antibodies conjugated to horseradish peroxidase $(1: 10,000)$ for $1 \mathrm{~h}$ at room temperature, and developed using an Electrochemiluminescence (ECL) kit (Thermo Fisher Scientific, Inc., Waltham, MA, USA). Anti-HIWI, anti-MMP-2, anti-MMP-9, anti-p21, anti-cyclin D1 (Beijing Biosea Biotechnology Co., Ltd., Beijing, China), anti-Bcl-2, and anti-Bax antibodies (Signalway Antibody, College Park, $\mathrm{MD}$, USA) were used to detect the respective proteins. An anti- $\beta$-actin antibody (Beijing Biosea Biotechnology Co., Ltd.) was used as a control for equal sample loading.

MTT assay. U251 and A172 cells $\left(5 \times 10^{3}\right)$ were incubated in 96-well plates, with each well containing $100 \mu 1$ of medium. The cells were divided into the following groups: i) control; ii) scrambled siRNA (cells transfected with control siRNA); and iii) HIWI siRNA group (cells transfected with HIWI siRNA). The transfection of siRNAs was performed the day after plating when cells were $\sim 40 \%$ confluent. The rate of cellular proliferation was measured $48 \mathrm{~h}$ after treatment. To each well $15 \mu 1$ of $5 \mathrm{mg} / \mathrm{ml} \mathrm{3-(4,5-dimethylthi-}$ azol-2-yl)-2,5-diphenyltetrazolium bromide (MTT) (Sigma, St. Louis, MO, USA) was added. After $4 \mathrm{~h}, 150 \mu \mathrm{l}$ of DMSO were added to the MTT-treated wells, and the absorption at $490 \mathrm{~nm}$ was determined using a spectrometer. Each experimental condition was performed in triplicate.

Colony formation. Cells in each group described above were trypsinized, counted, and seeded for colony formation assays in 12-well plates at 100 cells/well. During colony growth, the culture media were replaced every 3 days. A colony was counted only if it contained $>50$ cells, and the number of colonies was counted 14 days after seeding. The colony formation rate was calculated using the equation: colony formation rate $=($ number of colonies/number of seeded cells $) \times 100 \%$. Each treatment was performed in triplicate.

Flow cytometric analysis of apoptosis. U251 and A172 cells at $40 \%$ confluence were transfected with negative control or HIWI siRNA. At $48 \mathrm{~h}$ post-transfection, cells were harvested by trypsinization. Cells $\left(1 \times 10^{5}\right)$ were washed with PBS, and resuspended in $250 \mu 1$ of binding buffer containing $5 \mu \mathrm{l}$ Annexin V-FITC and $5 \mu 1$ propidium iodide (PI) (BD Pharmingen, San Diego, CA, USA). After incubation for $20 \mathrm{~min}$ at room temperature in the dark, another $400 \mu \mathrm{l}$ of binding buffer was added, and the samples were analyzed immediately using FACSCalibur. Flow cytometry data were analyzed using FlowJo (BD Pharmingen).

Flow cytometric analysis of the cell cycle. Control U251 and A172 cells, and those treated with HIWI or control siRNA $(50 \mathrm{nM})$ for $48 \mathrm{~h}$ were harvested, washed twice with cold PBS, and fixed with $5 \mathrm{ml}$ of ice-cold $70 \%$ ethanol overnight. The cells were then incubated with $1 \mathrm{mg} / \mathrm{ml}$ RNase A, $1 \%$ Triton X-100 (both from Sigma), and $50 \mu \mathrm{g} / \mathrm{ml}$ PI at $4^{\circ} \mathrm{C}$ overnight, and the DNA content was determined using flow cytometry (BD Pharmingen). The data were then analyzed using MLT software (Becton-Dickinson, San Jose, CA, USA).

Transwell invasion and migration assays. Cell invasion and migration assays were performed using a Transwell system that incorporated a polycarbonate filter membrane with a diameter of $6.5 \mathrm{~mm}$ and pore size of $8 \mu \mathrm{m}$ (Corning Costar), according to the manufacturer's protocol. To assess invasion, filters were pre-coated with $50 \mu 1$ of mixed Matrigel (Matrigel:DMEM = 1:5; BD Biosciences, Franklin Lakes, NJ, USA). Cell suspensions $\left(1 \times 10^{5}\right)$ in serum-free culture media were added into the inserts, and each insert was placed in the well of a plate filled with culture media containing $10 \% \mathrm{FBS}$ as a chemoattractant. After $24 \mathrm{~h}$ incubation at $37^{\circ} \mathrm{C}$, the non-invasive cells were removed from the upper chamber by wiping with cotton-tipped swabs. Filters were fixed using methanol and glacial acetic acid (1:3) for $30 \mathrm{~min}$, and then stained with $3 \%$ crystal violet solution for $30 \mathrm{~min}$ at room temperature. Five fields of adherent cells in each well were photographed randomly under a Nikon-TE2000-U inverted fluorescence microscope, and counted using ImageJ software.

The same experimental design was used for migration experiments, except that filters were not precoated with Matrigel, and cells were counted after $8 \mathrm{~h}$ incubation. All experiments were performed in triplicate. 
A

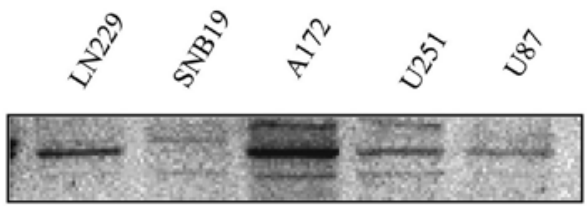

HIWI

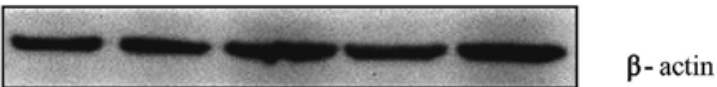

B

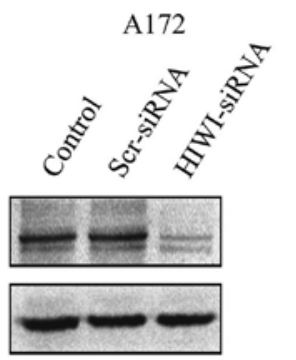

*
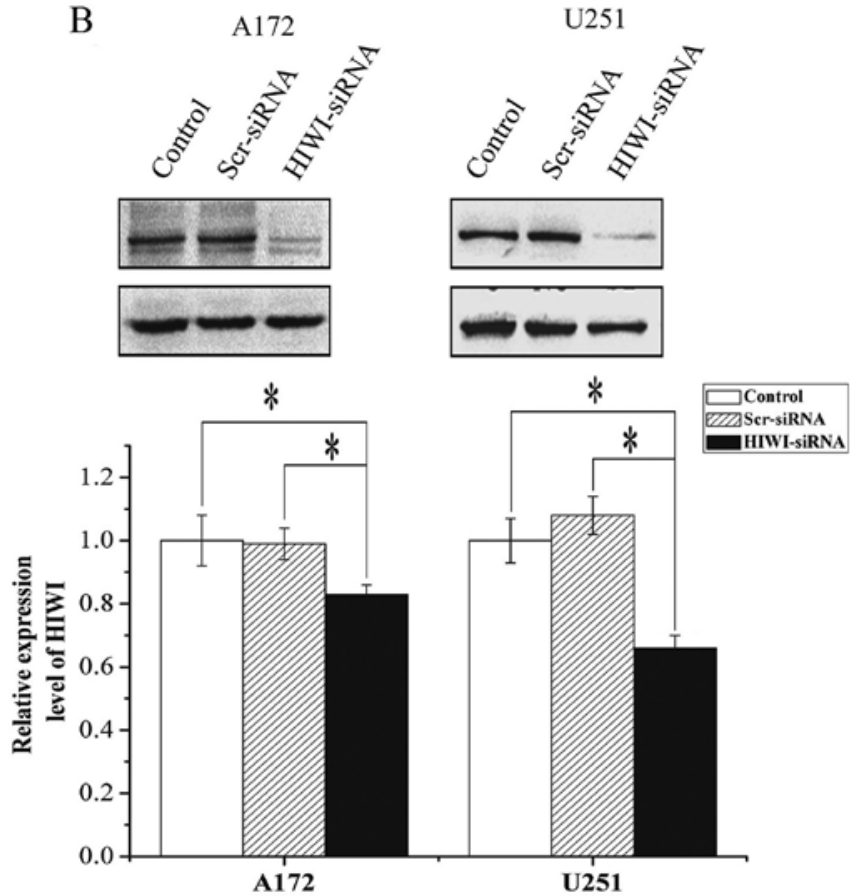

A172
A

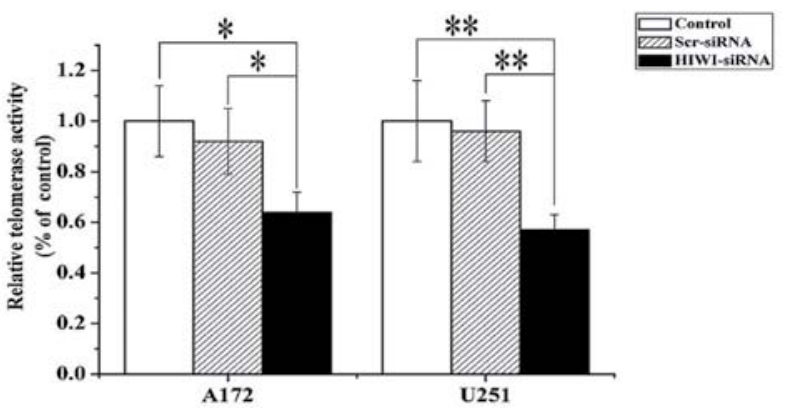

B

Control

Scr-siRNA

HIWI-siRNA
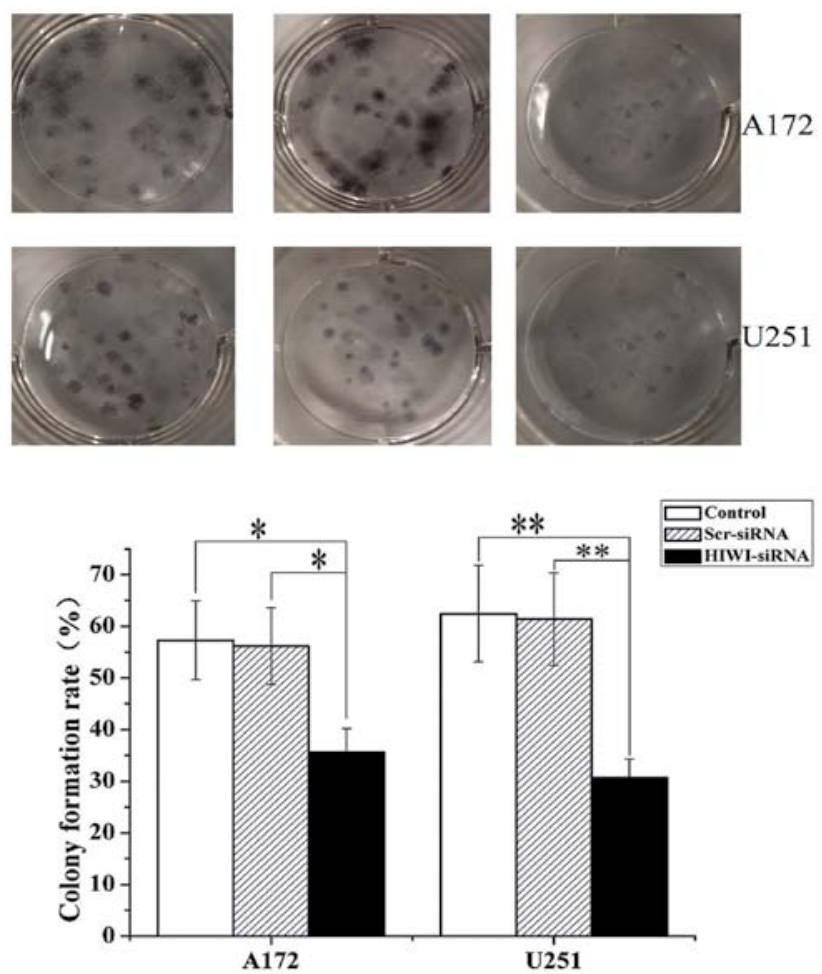

Figure 2. Effect of HIWI silencing on U251 and A172 cell proliferation. (A) The relative cell viability was measured using 3-(4,5-dimethylthiazol-2-yl)-2,5-diphenyltetrazolium bromide (MTT) assay. Control, scrambled siRNA, and HIWI siRNA groups were analyzed $48 \mathrm{~h}$ after treatment by MTT assay; cell proliferation was expressed as absorbance values. The results shown are representative of three independent experiments; histograms represent the mean, and error bars represent standard deviation (SD). (B) Detection of cell proliferation by plate colony formation assay in U251 and A172 cells. Representative photographs showing U251 and A172 cell colonies in 12-well plates. Cell colonies were scored visually, and counted using light microscopy. Data represent the mean $\pm \mathrm{SD}$ of three independent experiments. ${ }^{*} \mathrm{P}<0.05$ and $^{* *} \mathrm{p}<0.01$ in comparison with control group and scrambled siRNA group. The control group was untransfected, the scrambled siRNA group was transfected with control siRNA, and the HIWI siRNA group was transfected with HIWI siRNA.

The U251 glioma subcutaneous model was established described previously (17). The mice were randomly divided into three groups with six mice in each treatment group: HIWI siRNA group, scramble group and control group. A mixture of $20 \mu \mathrm{l}$ Lipofectamine 2000 and HIWI siRNA, nonsense siRNA $(20 \mathrm{nmol} / 1 \times 20 \mu \mathrm{l})$ or PBS mixture was injected into the xenograft model in a multisite injection manner. Treatment was conducted every 2 days, and the tumor volume was measured with a caliper every 2 days, using a formula (volume $=$ long diameter $\mathrm{x}$ short diameter ${ }^{2} / 2$ ) (18). After being observed for 28 days, the mice bearing xenograft tumors were sacrificed and the tumor tissues were removed for formalin fixation and the prepara- tion of paraffin embedded sections for immunohistochemical analysis. The mice were maintained at an animal facility under pathogen-free conditions. The handling of mice and experimental procedures were conducted in accordance with experimental animal guidelines.

Immunohistochemistry assay. The immunohistochemical staining was detected using streptavidin-biotin-horseradish peroxidase complex (SABC) compound (SABC kits; Wuhan 
A

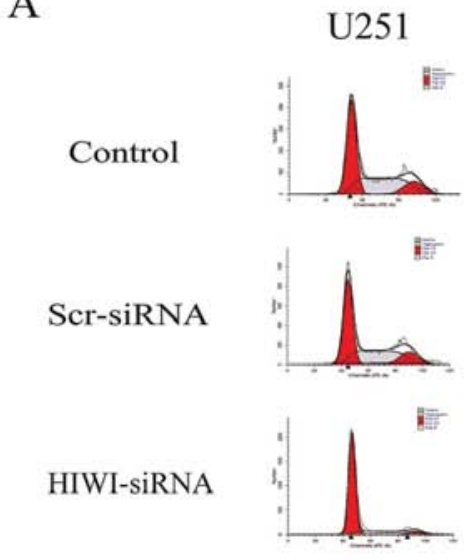

B

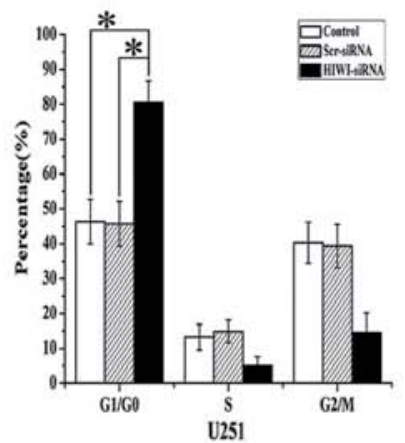

A172
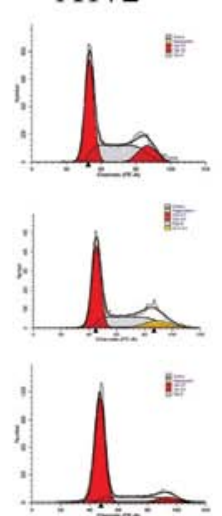

C
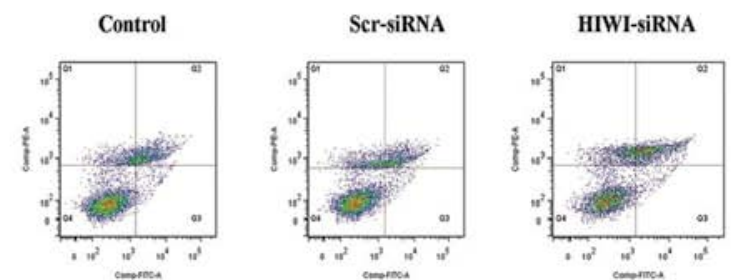

A172
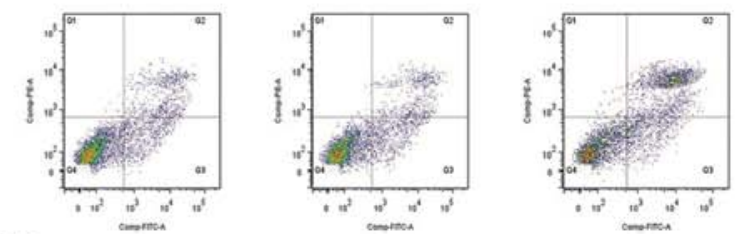

U251

D
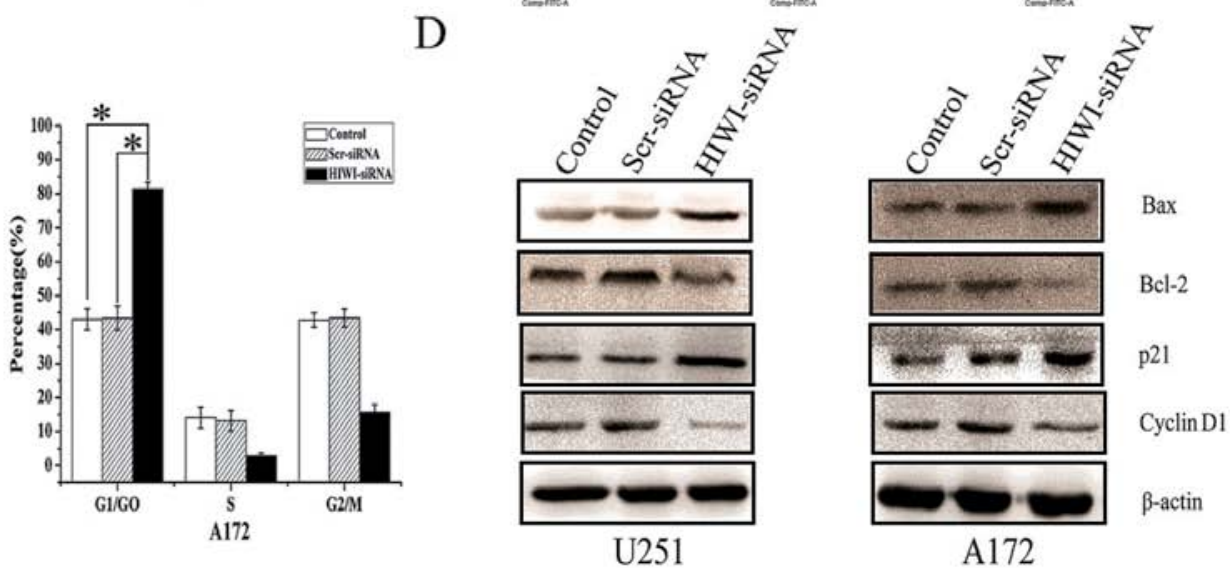

Figure 3. HIWI knockdown induced cell cycle arrest in G0/G1, induced apoptosis, and altered the expression of related proteins in glioma cell lines. (A) Silencing of HIWI by siRNA transfection affected cell cycle distribution in U251 and A172 glioma cancer cells. At 48 h post-transfection, DNA content was measured using propidium iodide (PI) staining by flow cytometry. (B) Knockdown of HIWI by RNAi in U251 and A172 cells induced cell cycle arrest in G0/G1 phase $48 \mathrm{~h}$ after transfection. ${ }^{\mathrm{P}}<0.05$ in comparison with control group and scrambled siRNA group. (C) For flow cytometry, control cells or those transfected with HIWI or scrambled siRNA were harvested $48 \mathrm{~h}$ after transfection, and stained with Annexin V-FITC and PI. Annexin V-FITC and PI double staining flow cytometry analysis showed that the cells transfected with HIWI siRNA exhibited significantly increased apoptosis $(42.71 \pm 3.14 \%, 31.46 \pm 2.53 \%)$ compared with normal cells $(23.43 \pm 1.68 \%, 19.82 \pm 1.45 \%)$ and cells treated with scrambled siRNA $(25.17 \pm 1.42 \%, 20.06 \pm 1.36 \%)$. Data represent the mean \pm standard deviation (SD) of three independent experiments. ${ }^{* *} \mathrm{P}<0.01$ in comparison with control group and scrambled siRNA group. (D) Total cellular proteins were treated as described above, and harvested after $72 \mathrm{~h}$. Western blotting was then performed using antibodies against p21, cyclin D1, Bcl-2, and Bax; $\beta$-actin was used as a loading control.

Boster Biological Technology, Ltd., Hubei, China) according to the manufacturer's protocol. Briefly, after incubation with endogenous peroxidase by $3 \% \mathrm{H}_{2} \mathrm{O}_{2}$, the slides were blocked with normal goat serum for $30 \mathrm{~min}$ in a humidified chamber at room temperature and then incubated at $4^{\circ} \mathrm{C}$ overnight with appropriate primary antibody (dilution 1:100). Next, these slides were treated with biotinylated secondary antibody for $60 \mathrm{~min}$, and finally incubated with SABC-AP conjugate for $30 \mathrm{~min}$. Immunologic reaction was developed using 3,3'-diaminobenzidine (DAB substate kit; Beijing Zhongshan Golden Bridge Biotechnology Co., Ltd., Beijing, China). Positive cells were mounted by Image-Pro Plus5.0 software. Negative controls were performed by substituting the primary antibody with Tris-buffered saline.

Statistical analysis. Results were analyzed using SPSS software 17.0, and compared using one-way analysis of variance (ANOVA). Data are presented as mean \pm standard deviation (SD) of three or six independent experiments, as appropriate. $\mathrm{P}<0.05$ was considered to be statistically significant.

\section{Results}

Endogenous expression and knockdown of HIWI in glioma cells. HIWI expression was assessed in five glioma cell lines (LN229, SNB19, A172, U251, and U87) by western blotting. HIWI was expressed to a variable extent in the different cell lines (Fig. 1A). HIWI or scrambled siRNA was then transiently transfected into $\mathrm{U} 251$ and A172 cells. After $48 \mathrm{~h}$, the HIWI protein levels were quantified with western blotting. Although the $\beta$-actin internal control revealed equal loading between the three groups, the expression of HIWI in U251 or A172 cells treated with HIWI siRNA was reduced by $\sim 80$ or $65 \%$, respectively, compared with both untreated and negative siRNA-transfected cells.

Silencing HIWI inhibited cell viability in vitro. To determine whether HIWI functions as an oncogene, we examined the effects of HIWI knockdown on glioma cell proliferation. Cells were transfected with HIWI siRNA, and the number of viable cells was determined by MTT assay $48 \mathrm{~h}$ after transfection. As shown in Fig. 2A, HIWI siRNA significantly decreased 

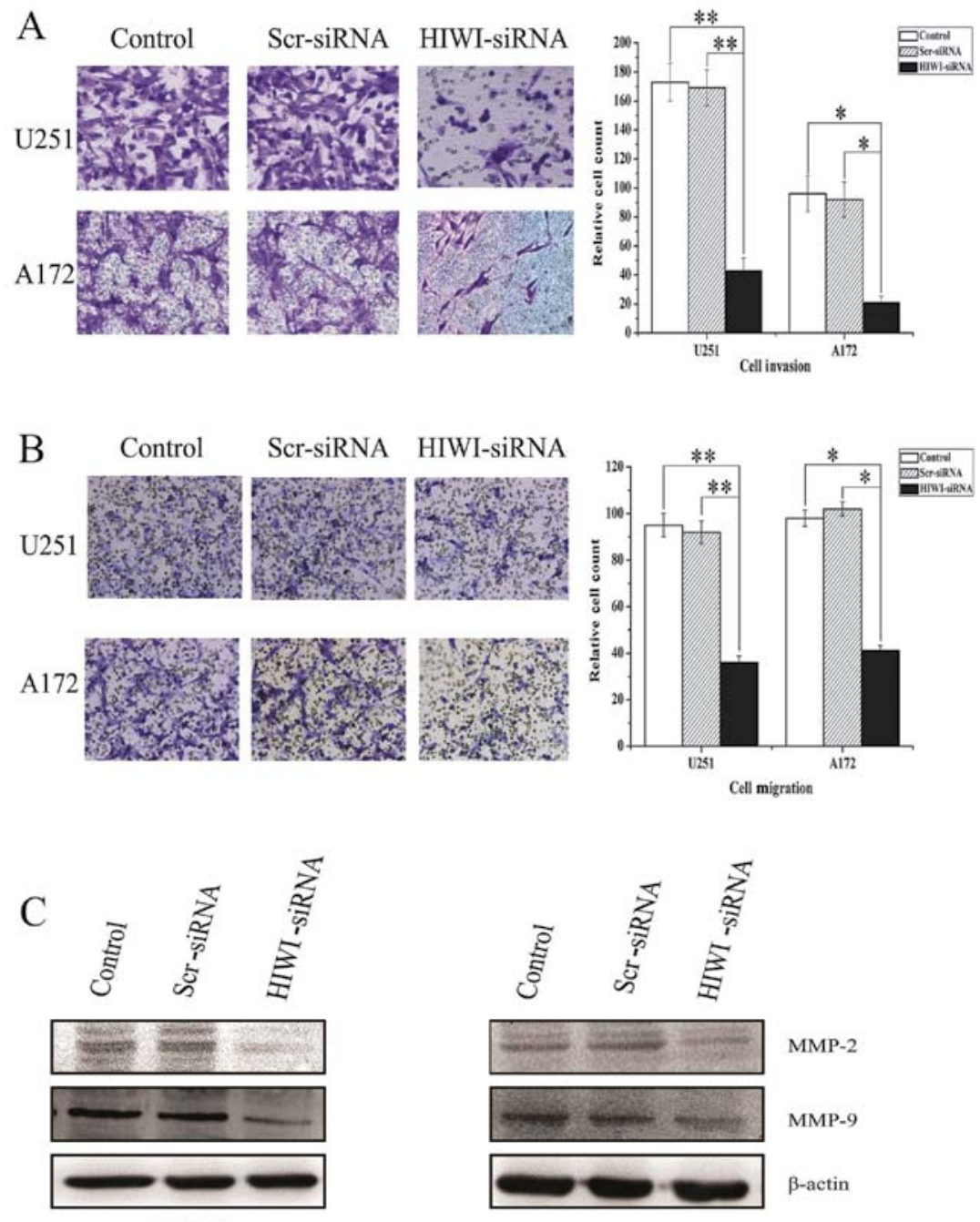

$\mathrm{U} 251$

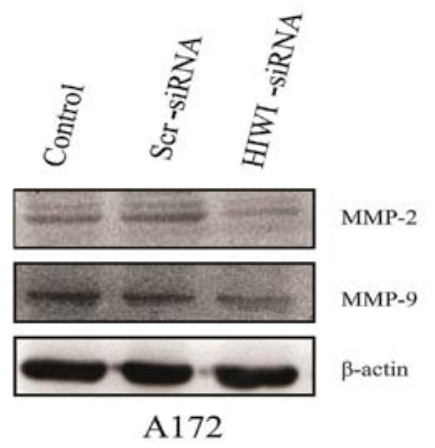

Figure 4. Downregulation of HIWI expression inhibited glioma cell migration and invasion. Transwell (A) invasion and (B) migration assays were performed to assess the invasive and migratory capacity, respectively, of control A172 and U251 or those transfected with scrambled or HIWI siRNA. (C) Western blotting showed that the expression of MMP-2 and MMP-9 was decreased significantly in HIWI siRNA-transfected U251 and A172 cells compared with control cells or those transfected with scrambled siRNA. ${ }^{*} \mathrm{P}<0.05$ and ${ }^{* *} \mathrm{p}<0.01$ in comparison with control group and scrambled siRNA group.

the percentage of viable cells in both U251 and A172 cell lines. These results confirmed that expression level of HIWI modulated cell growth, and that knockdown of HIWI exerted anti-proliferative effects.

To further assess the effects of HIWI on the growth of glioma cells, a colony formation assay was performed. As is shown in Fig. 2B, the number of colonies formed from U251 and A172 cells transfected with HIWI siRNA was significantly lower than untreated cells or those transfected with control siRNA. This suggests that knockdown of HIWI suppressed colony formation in U251 and A172 cells. These results were consistent with data obtained from the MTT assay, and further suggested that HIWI might be an oncogene.

Suppression of HIWI induced cell cycle arrest in G0/G1 phase, and altered the expression of cell cycle-related proteins in U25I and A172 cells. To further elucidate the growth suppressive effects of silencing HIWI in U251 and A172 cells, we performed cell cycle distribution analysis by flow cytometry $48 \mathrm{~h}$ after transfection with HIWI siRNA. As shown in Fig. 3A and B, the number of cells in G1 phase increased, but the number of cells in S phase decreased sharply after transfection with HIWI siRNA. To determine the molecular mechanism behind the inhibition of cell cycle after the downregulation of HIWI, we assessed the expression of important cell cycle regulatory proteins by western blotting. The expression of cyclin D1 decreased, whereas the expression of p21 increased in U251 and A172 cells that had been transfected with HIWI siRNA (Fig. 3D).

Knockdown of HIWI expression increased apoptosis in glioma cells by inducing Bcl-2 and Bax expression. We next determined whether the decrease in cell viability caused by HIWI knockdown was associated with increased apoptosis. The number of apoptotic untreated cells or those transfected with negative control or HIWI siRNA was assessed using Annexin V-FITC and PI labeling followed by flow cytometry. As shown in Fig. 3C, the number of late apoptotic cells was increased significantly $48 \mathrm{~h}$ after transfection with HIWI siRNA. In addition, the expression of Bcl-2 decreased, and Bax increased (Fig. 3D). 
A

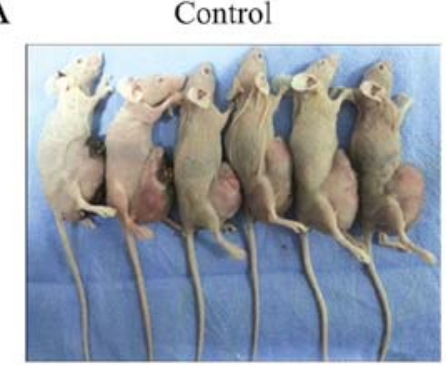

B

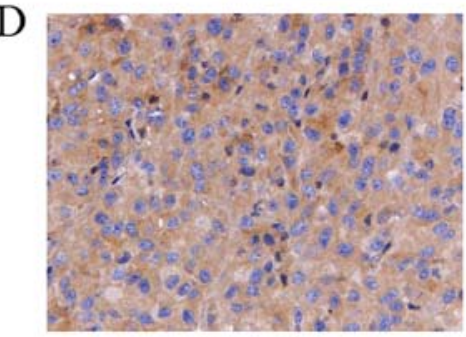

Control

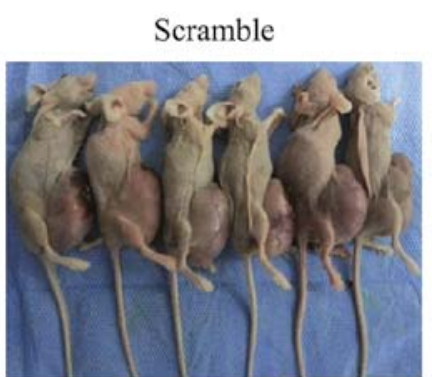

C
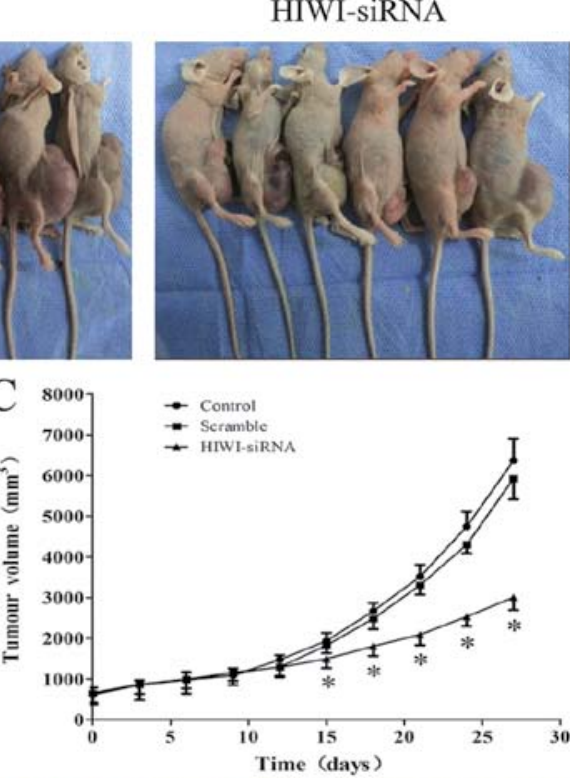

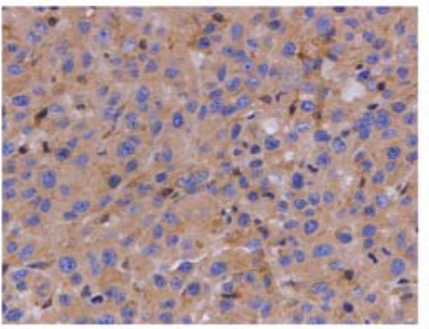

Scramble

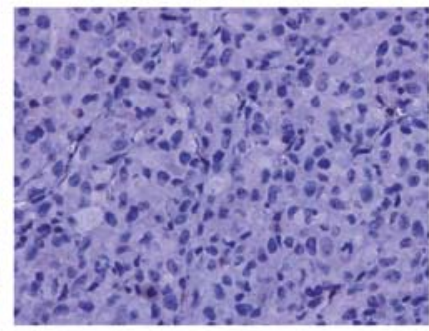

HIWI-siRNA

Figure 5. Reduced HIWI suppresses glioblastoma growth in the U251 xenograft tumor model. (A and B) Mice and tumor samples from the control group, the scrambled siRNA group and the HIWI siRNA group. (C) Tumor growth of three groups in proliferation assay in vivo. Downregulation of HIWI expression efficiently decreased tumor growth in vivo. ${ }^{*} \mathrm{P}<0.05$ in comparison with control group and scrambled siRNA group. (D) Representative photomicrographs of immunohistochemistry for HIWI, on xenograft tumor sections obtained from the control group, the scrambled siRNA group and the HIWI siRNA group. The levels of HIWI were suppressed in U251 xenograft tumors of the HIWI siRNA.

Downregulation of HIWI expression inhibited the migration and invasion of glioma cells in vitro. Transwell assays were performed to determine whether HIWI expression affected the migration and invasion of malignant glioma cells. Because the cells in tumor tissues exist in a three-dimensional environment, migration and invasion behavior is more complicated than in cell culture plates. Therefore, we used Transwell chambers to mimic the three-dimensional environment of tumor cells to assess the migration and invasion behavior of cells. As is shown in Fig. 4A, downregulation of HIWI reduced the number of migratory cells significantly compared with the scrambled siRNA and untreated control groups (Fig. 4B). Consistent with this, decreased invasion of U251 and A172 cells was also measured in the Matrigel Transwell assay after HIWI knockdown (Fig. 4C). Taken together, these results suggest that HIWI plays a vital role in the migration and invasion of glioma cells. To further explore the molecular mechanism by which HIWI promotes cellular migration and invasion, we measured the levels of matrix metalloproteinases (MMPs), which regulate these processes. Western blotting showed that the levels of MMP-2 and MMP-9 were reduced in U251 and A172 cells after HIWI knockdown (Fig. 4D).

Reduction of HIWI inhibits glioma growth in vivo. To explore the effect of HIWI activity on tumor growth in vivo, we employed a xenograft mouse model using U251 glioma cells treated with HIWI siRNA. On day 15, the tumor size of the HIWI siRNA group started to reach statistical significance compared with control groups $(\mathrm{p}<0.05)$. At the termination of the study, there was a marked reduction in tumor mass between the HIWI siRNA group and the control groups (Fig. 5A-C). After observation for 28 days, tumor samples were dissected from mice, and paraffin-embedded sections were prepared for immunohistochemical assay. Similar to the results obtained from the in vitro study, the expression of HIWI in tumor specimens of the HIWI siRNA group was highly downregulated (Fig. 5D).

\section{Discussion}

HIWI, a human homolog of the PIWI family that is important for stem cell self-renewal, is expressed highly in a variety of human cancers. Some studies have demonstrated that HIWI plays a key role in the development of tumors in cervical (19), colon (20), and liver cancer (21). Sun et al (15) also found that HIWI was expressed specifically in glioma tissues and cell lines, but its role and molecular mechanisms related to the development of glioma remain unclear. In this study, we first confirmed the expression of HIWI in glioma cell lines. To explore whether HIWI plays a significant role in the tumorigenesis and develop- 
ment of glioma, we next knocked down its expression in U251 and A172 glioma cells using RNAi. Inhibiting HIWI expression significantly decreased cell proliferation, increased apoptosis, and induced cell cycle arrest at G0/G1. We determined that decreased HIWI expression was accompanied by decreased migration and invasion of U251 and A172 cells, as measured by Transwell assays. Finally, we found that reduction of HIWI inhibited glioma growth in vivo.

A main feature of cancer is uncontrolled cell growth and proliferation. Previous studies revealed that cell proliferation is inhibited following the silencing of HIWI in human gastric cancer and lung cancer tumor stem cells $(13,22)$. This was due to inducing cell cycle arrest at $\mathrm{G} 2 / \mathrm{M}$ phase and inducing apoptosis, respectively, after the suppression of HIWI expression. However, a different study found that cell proliferation was reduced dramatically and apoptosis was induced in KG1 cells, a human leukemia cell line that lacks HIWI expression, when HIWI was expressed transiently (23). Our study revealed that cell proliferation was decreased significantly after knockdown of HIWI in glioma cells, which was confirmed by MTT and colony formation assays. The decline in cell proliferation was explained by both increased apoptosis and cell cycle arrest, which is partly consistent with previous studies. We speculated that HIWI could exert varying effects in a cell- and tissue-specific manner. Therefore, further studies will be needed to clarify these mechanisms.

Inhibiting cell cycle progression in cancer cells is considered to be one of the most effective strategies for regulating tumor growth. The shift from a dormant quiescent stage (G0) to an actively growing state is a prerequisite for entry into the cell cycle in most cells, and a crucial step for cancer cells (24). The cyclins and cyclin-dependent kinase (CDK) inhibitors strictly regulate cell cycle progression. The first of these proteins to be identified and cloned was p21. p21 is a universal cell cycle inhibitor that binds to cyclin-CDK complexes and proliferating cell nuclear antigen, thereby inducing cell arrest at G1 and blocking cell entry into $\mathrm{S}$ phase (25). Therefore, we assessed the relationships between G1 arrest and cell cycle related regulatory proteins, including p21 and cyclin D1, after HIWI knockdown. Data revealed that HIWI-mediated G1 arrest in glioma cancer cells was linked with p21. The upregulation of p21 enhances the formation of complexes with the G1/S CDKs and cyclins, inhibiting their activities and promoting apoptosis. Future studies should examine the underlying mechanisms by which HIWI upregulates p21 expression and modulates the downstream pathways.

Oncogenesis is also associated with abnormal apoptosis. As such, tumorigenesis can be defined by the imbalance between cell proliferation and apoptosis. Many studies have suggested that cell cycle arrest and apoptosis may be linked $(26,27)$. In addition, it was reported that the overexpression of $\mathrm{p} 21$ resulted in increased Bax expression and the induction of apoptosis. Liang et al demonstrated that silencing HIWI induced apoptosis in lung cancer stem cells (13). In this study, data showed that silencing HIWI also stimulated U251 and A172 apoptosis, increased Bax expression, and decreased Bcl-2 expression.

In addition to the function of HIWI as a potent growth regulator, another study revealed that low levels of expression of HIWI led to a significant decrease in the migration and invasion of hepatocellular carcinoma cells (16). The invasion of glioma cells into brain tissue is a pathologic hallmark of World Health Organization (WHO) grade II-IV gliomas, which contributes significantly to the failure of current treatments (28). Glioma cell invasion involves the attachment of tumor cells to the extracellular matrix (ECM), the degradation of ECM components, and the subsequent penetration into adjacent brain structures (29). MMPs are a family of extracellular zinc-dependent endopeptidases that selectively cleave the protein components of the ECM (30). Previous reports suggested that the expression of gelatinase-A (MMP-2) and gelatinase-B (MMP-9) play an important role in the invasion or metastasis of neoplastic tissue, since these are critical factors in basement membrane degradation (31). Numerous studies have demonstrated that malignant glioma cells secrete MMPs to facilitate their migration and invasion $(32,33)$. Therefore, MMPs may be involved in glioma cell invasion. A better understanding of the mechanisms of glioma invasion will facilitate the development of therapeutic strategies to decrease glioma invasion. Our data suggest that the silencing of HIWI inhibited glioma cell invasion and migration by suppressing MMP-2 and MMP-9 production. The activation of some signaling pathways can promote the expression of MMP-2 and MMP-9; however, it is unclear whether HIWI plays a role in these signaling pathways. The potential relationship between HIWI and MMPs will be assessed in future studies.

In summary, these findings suggest that HIWI plays an important role in the development of human glioma. Silencing HIWI expression could inhibit glioma cell proliferation, attenuate migration and invasion, promote apoptosis, and cause cell cycle arrest. This suggests that targeting HIWI could be a promising treatment modality for glioma in the future.

\section{Acknowledgements}

The study was supported by the Scientific and Technological Project of Tianjin Bureau of Public Health (11KG115), the National Key disciplines Fund of the Ministry of Health of the People's Republic of China, the Foundation of Tianjin Science and Technology Committee (12ZCDZSY17700, 14JCZDJC35600).

\section{References}

1. Maher EA, Furnari FB, Bachoo RM, et al: Malignant glioma: genetics and biology of a grave matter. Genes Dev 15: 1311-1333, 2001.

2. Furnari FB, Fenton T, Bachoo RM, et al: Malignant astrocytic glioma: genetics, biology, and paths to treatment. Genes Dev 21: 2683-2710, 2007.

3. Stupp R, Roila F; ESMO Guidelines Working Group: Malignant glioma: ESMO clinical recommendations for diagnosis, treatment and follow-up. Ann Oncol 20 (Suppl 4): S126-S128, 2009.

4. Argyriou AA and Kalofonos HP: Molecularly targeted therapies for malignant gliomas. Mol Med 15: 115-122, 2009.

5. Cox DN, Chao A, Baker J, Chang L, Qiao D and Lin H: A novel class of evolutionarily conserved genes defined by piwi are essential for stem cell self-renewal. Gen Dev 12: 3715-3727, 1998.

6. Sasaki T, Shiohama A, Minoshima S and Shimizu N: Identification of eight members of the Argonaute family in the human genome. Genomics 82: 323-330, 2003.

7. Hutvagner G and Simard MJ: Argonaute proteins: key players in RNA silencing. Nat Rev Mol Cell Biol 9: 22-32, 2008.

8. Siddiqi S, Terry M and Matushansky I: Hiwi mediated tumorigenesis is associated with DNA hypermethylation. PLoS One 7: e33711, 2012 
9. Wang Y, Liu Y, Shen X, et al: The PIWI protein acts as a predictive marker for human gastric cancer. Int J Clin Exp Pathol 5: 315-325, 2012

10. Grochola LF, Greither T, Taubert H, et al: The stem cell-associated Hiwi gene in human adenocarcinoma of the pancreas: expression and risk of tumour-related death. Br J Cancer 99: 1083-1088, 2008.

11. Li S, Meng L, Zhu C, et al: The universal overexpression of a cancer testis antigen hiwi is associated with cancer angiogenesis. Oncol Rep 23: 1063-1068, 2010.

12. Zeng Y, Qu LK, Meng L, et al: HIWI expression profile in cancer cells and its prognostic value for patients with colorectal cancer. Chin Med J (Engl) 124: 2144-2149, 2011.

13. Liang D,Fang Z, Dong M, et al:Effect of RNA interference-related HiWi gene expression on the proliferation and apoptosis of lung cancer stem cells. Oncol Lett 4: 146-150, 2012.

14. Liu C, Qu L, Dong B, et al: Combined phenotype of 4 markers improves prognostic value of patients with colon cancer. Am J Med Sci 343: 295-302, 2012.

15. Sun G, Wang Y, Sun L, et al: Clinical significance of Hiwi gene expression in gliomas. Brain Res 1373: 183-188, 2011

16. Zhao YM, Zhou JM, Wang LR, et al: HIWI is associated with prognosis in patients with hepatocellular carcinoma after curative resection. Cancer 118: 2708-2717, 2012.

17. Zhou X, Ren Y, Moore L, et al: Downregulation of miR-21 inhibits EGFR pathway and suppresses the growth of human glioblastoma cells independent of PTEN status. Lab Invest 90: 144-155, 2010.

18. Hahnfeldt P, Panigrahy D, Folkman J and Hlatky L: Tumor development under angiogenic signaling: a dynamical theory of tumor growth, treatment response, and postvascular dormancy. Cancer Res 59: 4770-4775, 1999.

19. Liu WK, Jiang XY and Zhang ZX: Expression of PSCA, PIWIL1 and TBX2 and its correlation with HPV16 infection in formalin-fixed, paraffin-embedded cervical squamous cell carcinoma specimens. Arch Virol 155: 657-663, 2010.

20. Li L, Yu C, Gao H and Li Y: Argonaute proteins: potential biomarkers for human colon cancer. BMC Cancer 10: 38, 2010.

21. Jiang J, Zhang H, Tang Q, Hao B and Shi R: Expression of HIWI in human hepatocellular carcinoma. Cell Biochem Biophys 61: 53-58, 2011.

22. Liu X, Sun Y, Guo J, et al: Expression of hiwi gene in human gastric cancer was associated with proliferation of cancer cells. Int J Cancer 118: 1922-1929, 2006
23. Sharma AK, Nelson MC, Brandt JE, et al: Human CD34(+) stem cells express the hiwi gene, a human homologue of the Drosophila gene piwi. Blood 97: 426-434, 2001.

24. Janssen A and Medema RH: Mitosis as an anti-cancer target. Oncogene 30: 2799-2809, 2011.

25. Cornils H, Kohler RS, Hergovich A and Hemmings BA: Human NDR kinases control G(1)/S cell cycle transition by directly regulating p21 stability. Mol Cell Biol 31: 1382-1395, 2011.

26. Ghate NB, Chaudhuri D, Sarkar R, et al: An antioxidant extract of tropical lichen, Parmotrema reticulatum, induces cell cycle arrest and apoptosis in breast carcinoma cell line MCF-7. PLoS One 8: e82293, 2013.

27. Nakada M, Nakada S, Demuth T, Tran NL, Hoelzinger DB and Berens ME: Molecular targets of glioma invasion. Cell Mol Life Sci 64: 458-478, 2007

28. Itoh Y, Palmisano R, Anilkumar N, Nagase H, Miyawaki A and Seiki M: Dimerization of MT1-MMP during cellular invasion detected by fluorescence resonance energy transfer. Biochem J 440: 319-326, 2011.

29. Kargiotis O, Chetty C, Gondi CS, et al: Adenovirus-mediated transfer of siRNA against MMP-2 mRNA results in impaired invasion and tumor-induced angiogenesis, induces apoptosis in vitro and inhibits tumor growth in vivo in glioblastoma. Oncogene 27: 4830-4840, 2008.

30. Zhou X, Ma L, Li J, Gu J, Shi Q and Yu R: Effects of SEMA3G on migration and invasion of glioma cells. Oncol Rep 28 269-275, 2012

31. Munaut C, Noël A, Hougrand O, Foidart JM, Boniver J and Deprez M: Vascular endothelial growth factor expression correlates with matrix metalloproteinases MT1-MMP, MMP-2 and MMP-9 in human glioblastomas. Int J Cancer 106: 848-855, 2003

32. Yoshida D, Watanabe K, Noha M, Takahashi H, Teramoto A and Sugisaki Y: Anti-invasive effect of an anti-matrix metalloproteinase agent in a murine brain slice model using the serial monitoring of green fluorescent protein-labeled glioma cells. Neurosurgery 52: 187-197, 2003.

33. Bellail AC, Hunter SB, Brat DJ, Tan C and Van Meir EG: Microregional extracellular matrix heterogeneity in brain modulates glioma cell invasion. Int J Biochem Cell Biol 36: 1046-1069, 2004. 